\title{
Radiation Analysis and Characteristics of Conformal Reflectarray Antennas
}

\author{
Payam Nayeri, ${ }^{1}$ Fan Yang, ${ }^{1,2}$ and Atef Z. Elsherbeni ${ }^{1}$ \\ ${ }^{1}$ Electrical Engineering Department, The University of Mississippi, Oxford, MS 38677, USA \\ ${ }^{2}$ Electronic Engineering Department, Tsinghua University, Beijing 100084, China
}

Correspondence should be addressed to Payam Nayeri, pnayeri@olemiss.edu

Received 3 February 2012; Accepted 11 May 2012

Academic Editor: Sandra Costanzo

Copyright ( 2012 Payam Nayeri et al. This is an open access article distributed under the Creative Commons Attribution License, which permits unrestricted use, distribution, and reproduction in any medium, provided the original work is properly cited.

This paper investigates the feasibility of designing reflectarray antennas on conformal surfaces. A generalized analysis approach is presented that can be applied to compute the radiation performance of conformal reflectarray antennas. Using this approach, radiation characteristics of conformal reflectarray antennas on singly curved platforms are studied and the performances of these designs are compared with planar designs. It is demonstrated that a conformal reflectarray antenna can be a suitable choice for applications requiring high-gain antennas on curved platforms.

\section{Introduction}

Printed reflectarray antennas combine the favorable features of both printed arrays and parabolic reflectors and create a low-profile, low-mass, and low-cost antenna [1]. A reflectarray antenna imitates the conventional parabolic reflectors, with the added advantage of having a flat surface instead of the curved reflecting surface. Owing to their high-gain and low-loss characteristics, the reflectarray antenna has received considerable attention over the years. The surface of the reflectarray antenna consists of phase changing elements, which create the collimated beam and are usually distributed on a planar aperture.

Several applications require the use of conformal antenna technology. While the need for a conformal antenna system generally depends on the specific application, the advantages can usually be attributed to either mechanical (aerodynamic, hydrodynamic) or electrical benefits [2]. In terms of the mechanical advantages, a conformal antenna is designed according to a prescribed shape, which can be some part of an airplane, high-speed train, or other vehicle. The main purpose is to build an antenna that becomes integrated with the structure and causes less disturbance such as extra drag. The electrical advantages of conformal antennas are typically wide angle scanning capabilities. Conformal beam-scanning array antennas can achieve full azimuth and wide elevation coverage [3], which in most cases is not possible with planar designs.

In this paper, we investigate the feasibility of designing reflectarray antennas on conformal surfaces. In section II, a generalized analysis approach to compute the radiation pattern of conformal reflectarray antennas is presented. In section III, reflectarray antennas on singly curved conformal cylindrical platforms are studied and radiation performances of these designs are compared with planar designs. It is demonstrated in these studies that a conformal reflectarray antenna can be a suitable choice for a high-gain antenna where curved platforms are required.

\section{Radiation Analysis of Conformal Reflectarrays}

2.1. Radiation Pattern Calculation. The radiation pattern of a planar reflectarray antenna with thousands of elements can be computed quite efficiently using the classical array theory formulation with proper element excitation [4]. In a recent study, it was shown that the calculated radiation pattern using this approach shows a good agreement with full-wave simulations [5]. In particular, the main beam direction, beam width, and side lobe level match closely with those obtained using full-wave simulations. Therefore, the array 


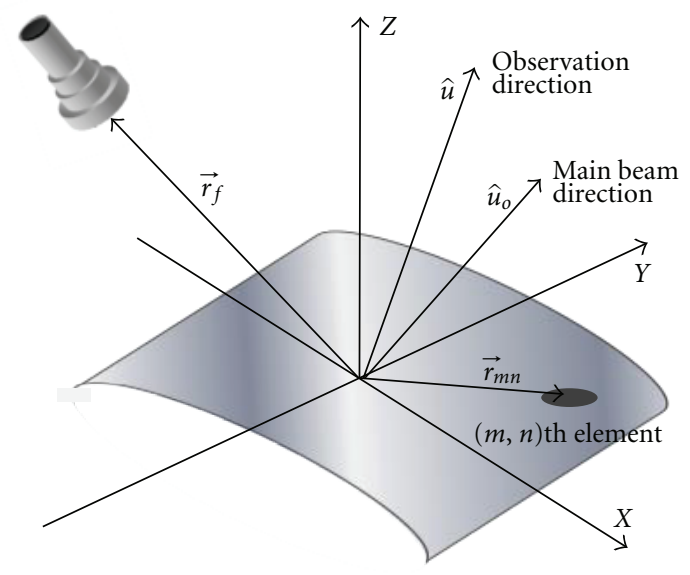

FIgURE 1: Coordinate system of the reflectarray antenna.

theory approach is used in this paper to study the radiation characteristics of conformal reflectarrays.

The formulation developed for planar reflectarray antennas can be generalized to analyze the performance of conformal reflectarrays. With the array theory formulation, as long as the elements on the conformal surface are correctly modeled with mutual coupling included, array summation can provide accurate results. Therefore, an important consideration in radiation analysis of conformal designs is the accurate modeling of element radiation characteristics. One should realize that the peak of the element pattern is normal to the local surface, so the peaks will not all point in the same direction for a conformal array [6]. Furthermore, a reflectarray antenna is functioning in the reflection mode, which is different from conventional antenna arrays [2]. Scattering properties of curved surfaces are more complicated than planar surfaces, and in general high order diffraction effects such as generation of creeping waves, and depolarization should be taken into account [7]. Nonetheless, if the conformal surface is not highly curved, edge diffraction is minimized, and hence microwave optic approaches are suitable for the analysis.

The radiation pattern of a conformal array with $M \times$ $N$ elements can be calculated using the following general formula:

$$
\begin{gathered}
\vec{E}(\hat{u})=\sum_{m=1}^{M} \sum_{n=1}^{N} \vec{A}_{m n}(\hat{u}) \cdot \vec{I}\left(\vec{r}_{m n}\right), \\
\hat{u}=\hat{x} \sin \theta \cos \varphi+\hat{y} \sin \theta \sin \varphi+\hat{z} \cos \theta,
\end{gathered}
$$

where $A$ is the element pattern vector function, $I$ is the element excitation vector function, and $\vec{r}_{m n}$ is the position vector of the $m n$th element. The geometry of the conformal reflectarray system is given in Figure 1.

A cosine $q_{e}$ model is usually used for the element pattern function $A$, which in general is not symmetric due to the surface curvature. Some highly curved conformal arrays may have a $\cos ^{1.5}$ behavior in the strong coupling plane and a $\cos ^{1}$ behavior in the weak coupling plane [7]. As discussed earlier, in our studies the conformal reflectarray surface is not highly curved, therefore each unit cell can be approximated with a planar surface that is tangential to the conformal surface. The element pattern can then be approximated with a symmetric $\cos ^{1}$ model with its peak normal to the unit cell surface. Therefore, the important considerations for element pattern modeling are to (1) determine the exact angle of incidence for each element that controls the receive mode element pattern and (2) determine the orientation of the local cell coordinates with respect to the global coordinates which controls the transmit mode element pattern.

The transmit element pattern function can be written as

$$
A_{m n}(\theta, \varphi) \approx \cos ^{q_{e}} \theta_{\text {local }} \cdot e^{j k\left(\vec{r}_{m n} \cdot \hat{u}\right)} .
$$

In comparison with planar designs [5], this function takes into account the effect of surface curvature on the transmit element pattern by using local element coordinates for each radiating element.

The element excitation function $I$ is determined by both the incident field and element property [4]. By approximating the feed horn pattern function using a cosine $q_{f}$ model and taking into account the Euclidian distance between the feed horn and the element, the illumination of the aperture can be obtained. The element receive pattern is essentially part of the element excitation function, which determines the reflected power from the element based on the angle of excitation and element pattern shape, that is,

$$
\left|\Gamma_{m n}\right|=\cos ^{q_{e}} \theta_{e}(m, n) .
$$

While the function here is similar to the planar case, the difference is that for conformal reflectarrays the element angle $\theta_{e}$ depends on both the element location and the local surface orientation.

With these approximations, the radiation pattern of a conformal reflectarray antenna can be simplified to the scalar form

$$
\begin{aligned}
E(\theta, \varphi)= & \sum_{m=1}^{M} \sum_{n=1}^{N} \cos ^{q_{e}} \theta_{\text {local }} \cos ^{q_{e}} \theta_{e}(m, n) e^{j \phi_{m n}} \\
& \times \frac{\cos ^{q_{f}} \theta_{f}(m, n)}{\left|\vec{r}_{m n}-\vec{r}_{f}\right|} e^{-j k\left(\left|\vec{r}_{m n}-\vec{r}_{f}\right|-\vec{r}_{m n} \cdot \hat{u}\right)} .
\end{aligned}
$$

In this equation $\theta_{f}$ is the spherical angle in the feed coordinates and $\vec{r}_{f}$ is the position vector of the feed. The required phase delay of the $m n$th element $\phi_{m n}$ is adjusted to set the main beam in the $\hat{u}_{o}$ direction.

The radiation pattern calculation method described here uses a conventional array summation technique with scalar function approximations. If the cross-polarization of the reflectarray is concerned, the polarization of the feed horn and elements need to be accounted for in a comprehensive model instead of the simplified cosine $q$ model presented above.

2.2. Gain Calculations. For an accurate calculation of the reflectarray antenna gain, it is imperative to determine the 
overall aperture efficiency of the antenna. Different kinds of efficiency factors are considered in conventional reflector antennas [8]. Amongst these efficiency factors however, the major terms that influence the reflectarray antenna gain are the spillover and taper efficiency [9].

The formulation presented in the previous section for calculating the radiation pattern, accurately takes into account the illumination of the aperture. In other words, the effect of taper efficiency is already taken into account when one calculates the radiation pattern directivity using (4). The spillover efficiency on the other hand requires some attention. By definition, spillover efficiency $\left(\eta_{s}\right)$ is the percentage of radiated power from the feed that is intercepted by the reflecting aperture. Mathematically this can be written as

$$
\eta_{s}=\frac{\iint_{\sigma} \vec{P}(\vec{r}) \cdot d \vec{s}}{\iint_{\Sigma} \vec{P}(\vec{r}) \cdot d \vec{s}},
$$

where both integrals are fluxes of the Poynting vector $P(r)$ through certain surface areas. The denominator is the total power radiated by the feed; therefore the integral is performed over the entire surface area of a sphere centered at the feed, denoted by $\Sigma$. The numerator is the power incident on the array aperture, thus it is evaluated over a portion $\sigma$ of the sphere, where $\sigma$ and the array aperture share the same solid angle with respect to the feed. With this model, the denominator in (5) will have a simple closed-form expression [9]. The numerator can be computed numerically for any conformal surface. This is generally the same approach one follows for spillover efficiency calculations of a planar reflectarray, however the difference here is that the integration has to be performed over a curved surface. The gain of the reflectarray antenna is then computed using

$$
G=\eta_{s} D
$$

Here $D$ is the maximum directivity of the reflectarray antenna which is defined as the maximum radiation intensity of the antenna over the average, and is calculated using (4). It should be noted that the gain calculations discussed here don't include the factors associated with the feed loss, reflectarray element loss, and polarization loss. However in most cases for reflectarrays with low-loss substrates, these factors don't contribute much to the overall loss of the system [10].

\section{Radiation Characteristics of Singly Curved Conformal Reflectarrays}

Low-profile printed antennas flush mounted on singly curved surfaces such as cylinders represent an important class of conformal arrays used in many radar and communication systems [11]. Cylindrical array antennas are mainly utilized for either full azimuth scanning or sector arrays covering a specific subtended angle. In this section, the radiation characteristics of reflectarrays on conformal sector cylindrical surfaces are investigated. The analysis of this

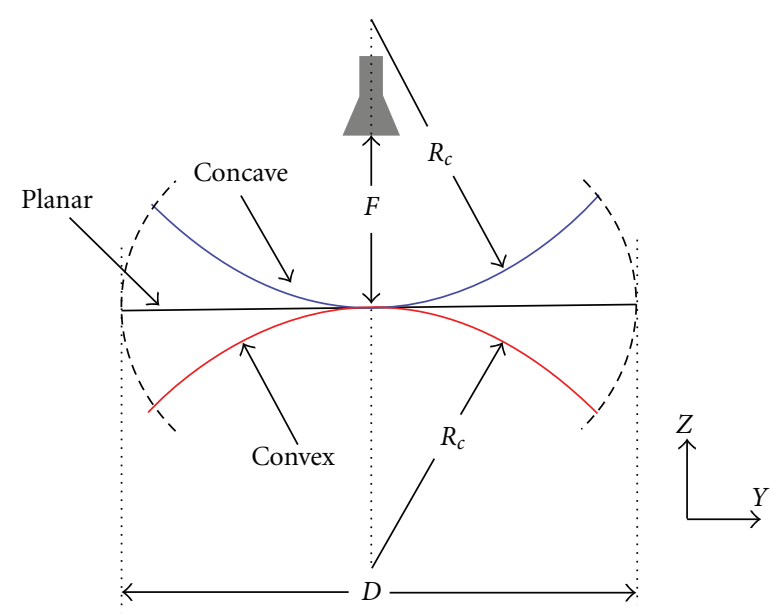

FIGURE 2: Cross-section of the conformal cylindrical reflectarrays systems.

singly curved canonical problem can provide an insight into many radiation characteristics of conformal reflectarrays. The cross-section of the conformal reflectarray system is shown in Figure 2, where concave and convex surfaces are compared with their planar counterpart with the same surface area. In this figure, $R_{c}$ is the radius of the cylinder for the conformal systems, and $D$ represents the physical aperture size.

It is important to point out that for conformal arrays with a broadside beam, using large element spacing (relative to wavelength) will result in generation of grating lobes in the visible range [7]. For element spacing in the order of halfwavelength, however, this error is substantially minimized, and this value is used for all numerical studies reported here. In addition, it should be noted that while, in general, there is a direct relation between the unit cell size (or element spacing) and the aperture curvature, for the half-wavelength unit cells used in this study, the maximum surface curvature of a single element is in the order of $1 / 20$ th of a radian, which ensures that a planar multifaceted approximation for the element performance is a suitable assumption.

3.1. Radiation Patterns of Cylindrical Reflectarrays. The numerical approach presented here is applied to the radiation analysis of sector reflectarray antennas mounted on cylindrical surfaces. For a meaningful comparison of the surface curvature effect, the feed properties and the physical aperture size are kept constant, and the only parameter that is changed is the cylinder radius. The reflectarray antennas have a $20 \lambda \times 20 \lambda$ aperture and are designed to generate a beam in the broadside direction. Ideal phasing elements are used and the element pattern is modeled as a $\cos ^{1}$ function with no azimuth dependence. The feed is prime focus, positioned with an $F / D=0.75$ and the power $q$ of radiation pattern model is 6.5.

The radiation pattern of the reflectarray systems in the curvature plane (yz-plane) is shown in Figure 3 for various 
cases. It should be noted that the radiation pattern in the $x z$ plane was similar to the planar case and is not shown here for brevity.

It can be seen that due to the effects of the conformal surface, the radiation patterns show a noticeable difference with planar designs $\left(R_{c} \rightarrow \infty\right)$. In particular, for the concave design, the side lobes are higher than the planar case. On the other hand, the convex designs show a lower side lobe level (SLL), but a much wider beamwidth. For both designs however, as the cylinder radius increases, the radiation patterns of the conformal designs approach that of a planar case. While this limit generally depends on the aperture size, for the cases studied here, the limit is almost $R_{c}=40 \lambda$.

3.2. Beamwidth and Side Lobe Level. The conformal cylindrical systems studied in the previous section were defined based on the size of the cylinder radius $\left(R_{c}\right)$. The aperture size $(D)$ however also plays an important role. For a fixed cylinder radius, a larger aperture means a wider subtended angle $\left(\alpha=D / R_{c}\right)$ and a higher level of surface curvature. To study this, effect we consider three different aperture sizes and compare the beamwidth and SLL of these designs. Similarly in all the designs, the feed $(q=6.5)$ is prime focus, and positioned with an $F / D$ of 0.75 . This would ensure that the aperture taper is identical for the different aperture sizes studied here. The normalized half-power beamwidth (HPBW) in the curvature plane as a function of cylinder radius is given in Figure 4 for different aperture sizes.

It can be seen that in comparison between the two conformal designs, a convex reflectarray shows a much wider beamwidth. In general, however, both conformal designs show defocusing effects, that is, a wider beamwidth in comparison with a planar design. This is mainly attributed to the fact that the elements on the conformal surface radiate in the outward (convex) or inward (concave) radial directions of the cylinder which defocus the main beam. It should be noted that for a concave design, an optimum position exists for the feed that can achieve maximum focusing. More discussion on this topic will be given in a subsequent section.

The change in SLL as a function of cylinder radius is given in Figure 5 for different aperture sizes. Comparisons of these results also show that in all cases as the aperture size increases, the SLL increases for the concave design and decreases for the convex design. In other words, the effect of the conformal surface on the radiation performance becomes more noticeable as the aperture size increases.

The results in Figures 4 and 5 indicate that the radiation performance of conformal cylindrical reflectarrays is a function of both cylinder radius and aperture size. However, it will be quite advantageous if one can determine the overall performance of the system based on a single conformal parameter. The subtended angle $\alpha$, takes into account the aperture size, $D$, which controls the gain, in addition to the cylinder radius, $R_{c}$, which controls the curvature of the conformal surface. As such, it can be a suitable measure to evaluate the performance of the system. Table 1 summarizes the change in radiation characteristics of some conformal designs with a fixed subtended angle of 1 radian.
TABLE 1: Change in Radiation Performance of Conformal Cylindrical Reflectarrays with $D / R_{c}=1$.

\begin{tabular}{llccc}
\hline Aperture size & Design & $10 \lambda \times 10 \lambda$ & $20 \lambda \times 20 \lambda$ & $30 \lambda \times 30 \lambda$ \\
\hline \multirow{2}{*}{ HPBW ratio } & Concave & 0.036 & 0.033 & 0.037 \\
& Convex & 0.077 & 0.080 & 0.083 \\
\hline \multirow{2}{*}{ SLL ratio $(\mathrm{dB})$} & Concave & 2.81 & 2.73 & 2.73 \\
& Convex & -0.40 & -0.25 & -0.38 \\
\hline
\end{tabular}

From these results, it can be seen that the ratio of the change in HPBW and SLL is almost identical for all three cases. Similar results were observed for other values of subtended angle. In other words, it is possible to correctly specify the radiation performance of a conformal reflectarray system based on the value of $\alpha$. This is quite advantageous from a system design view point, since for any available curved platform the radiation characteristics of the conformal reflectarray can be determined with only one conformal parameter. As an example for a $10 \%$ acceptable increase in HPBW in comparison with a planar design, $\alpha$ is equal to 1.62 and 1.13 radians for concave and convex designs, respectively, for any aperture size.

It is important to point out that the subtended angle $(\alpha)$ decreases as the cylinder radius increases. For the smallest cylinder radius, the aperture size increase corresponds to an increase in $\alpha$ from almost $\pi / 3$ to $\pi$. Larger apertures will not be practical here, since: (1) the elements cannot be properly excited (shadow region of feed), and (2) the pattern of the elements at the sector edges point far away from the broadside direction.

3.3. Gain and Bandwidth. The reflectarray antenna gain is directly proportional to its projected aperture. As such for a fixed aperture size, the projected aperture, and corresponding antenna gain of conformal designs will be slightly smaller than a planar design. For a $20 \lambda \times 20 \lambda$ aperture with a fixed value of $\alpha=1$, this will result in a gain reduction of 0.1 and $0.6 \mathrm{~dB}$ for concave and convex designs, respectively. While it is possible to mitigate this slight difference in antenna gain by increasing the aperture size, for the bandwidth comparisons here we will compare the normalized antenna gain as a function of frequency.

The bandwidth of the reflectarray antenna is affected by the phase error associated with the spatial delay as well as the bandwidth of elements [12]. While the element bandwidth generally depends on the design methodology [1], for this comparative study, we consider a frequency-independent reflection phase for the elements, that is, only the bandwidth limitation associated with spatial phase delay is taken into account. Figure 6 shows the normalized gain bandwidth of a $20 \lambda \times 20 \lambda$ reflectarray antenna with planar and conformal apertures ( $\alpha=1$ radian).

It can be seen that a concave design shows a notable improvement in gain bandwidth in comparison with the planar case. On the other hand, as expected, a convex design shows a reduction in bandwidth. It is also interesting to observe the bandwidth performance of these designs as 


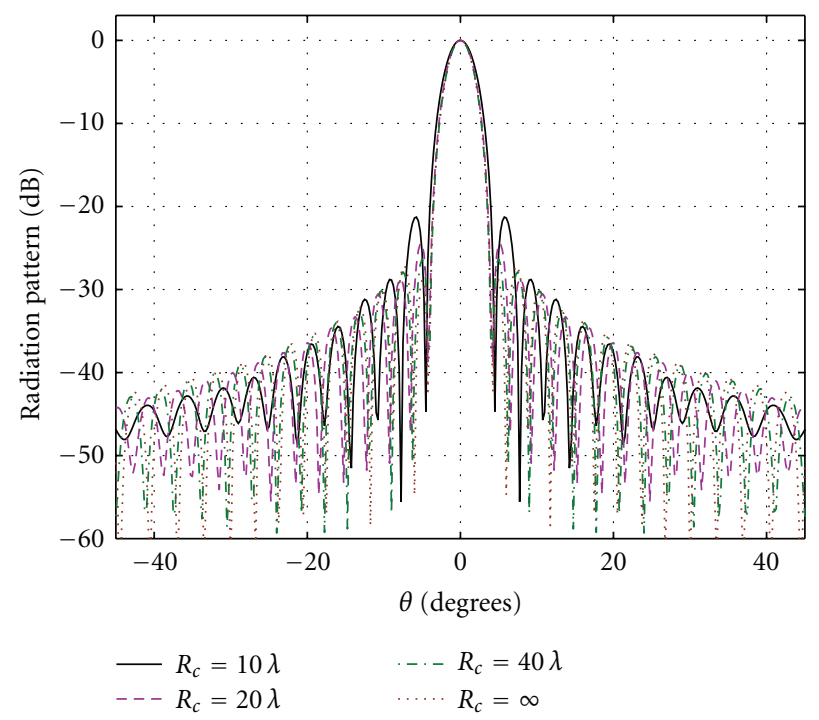

(a)

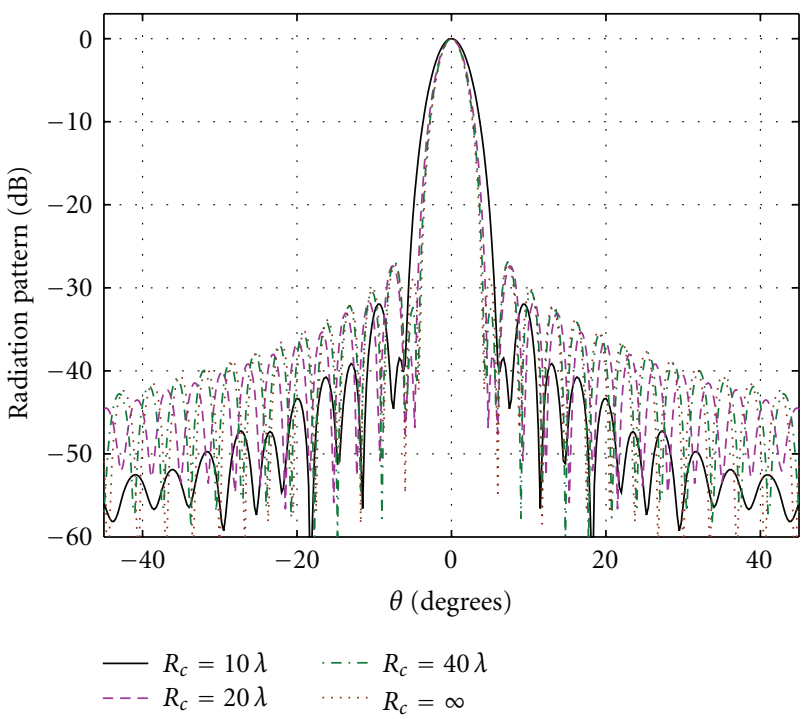

(b)

FIgURE 3: Radiation patterns of cylindrical reflectarrays: (a) concave, (b) convex.

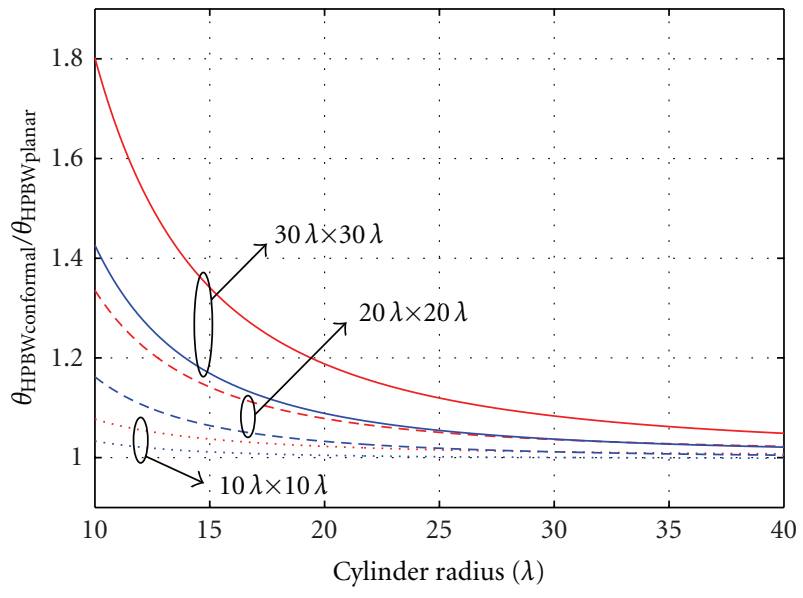

$\begin{array}{ll}\ldots . . .10 \lambda \times 10 \lambda \text { (Concave) } & --20 \lambda \times 20 \lambda \text { (Convex) } \\ \ldots . . .10 \lambda \times 10 \lambda \text { (Convex) } & -30 \lambda \times 30 \lambda \text { (Concave) } \\ \ldots--20 \lambda \times 20 \lambda \text { (Concave) } & -30 \lambda \times 30 \lambda \text { (Convex) }\end{array}$

FIGURE 4: Normalized HPBW in the curvature plane as a function of cylinder radius.

a function of cylinder radius. It should be noted here that in all designs the reflectarray aperture size is held constant $(20 \lambda \times 20 \lambda)$, and the change in cylinder radius corresponds to changing the subtended angle $\alpha$ from 2 to 0.5 radian. These results are shown in Figure 7.

For a convex design, the bandwidth of the reflectarray increases monotonously with $R_{c}$. For a concave design however, an optimum subtended angle exists where the antenna achieves the maximum bandwidth. For the design here, this optimum subtended angle is 0.67 radians.

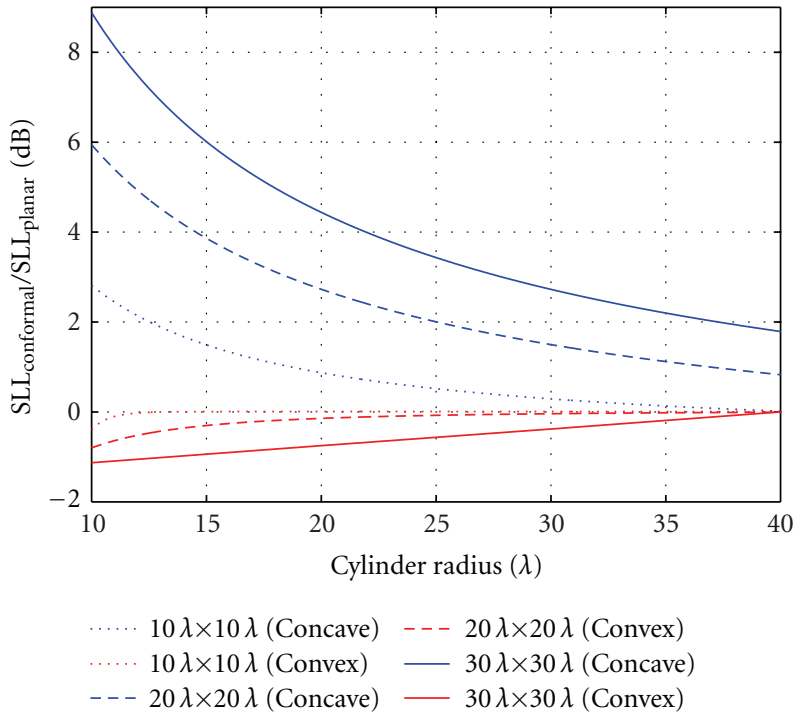

FIgURE 5: Normalized SLL in the curvature plane as a function of cylinder radius.

\section{Conclusion}

The feasibility of designing reflectarray antennas on conformal surfaces is investigated numerically. A generalized array theory formulation is presented to compute the radiation pattern of conformal reflectarray antennas. The radiation characteristics of concave and convex conformal reflectarrays on cylindrical surfaces are compared with planar designs. It is shown that in general, conformal reflectarrays will have a wider beamwidth than planar reflectarrays. The SLL depends on the platform, where concave designs show a higher SLL, while convex designs can achieve a lower SLL. In addition, 


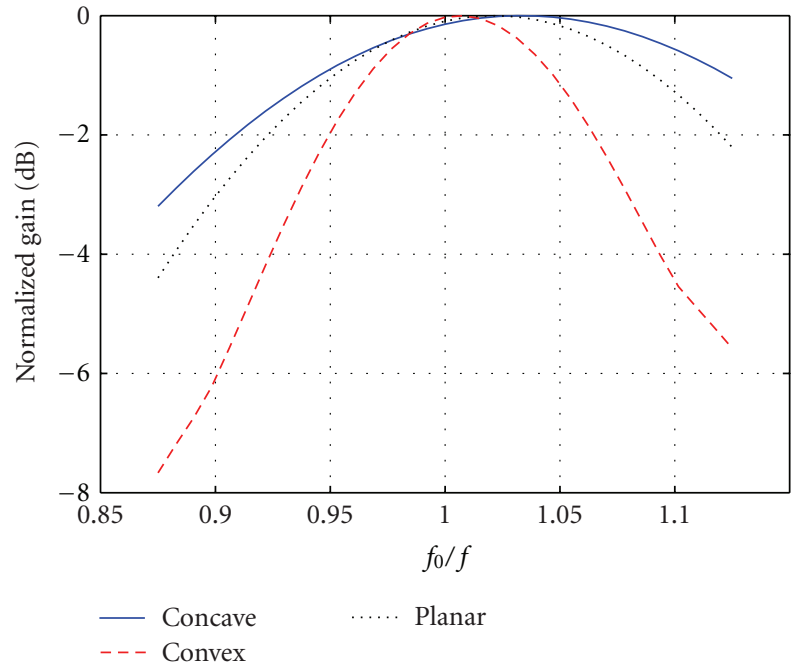

FIGURE 6: Normalized gain versus frequency for the reflectarray antennas.

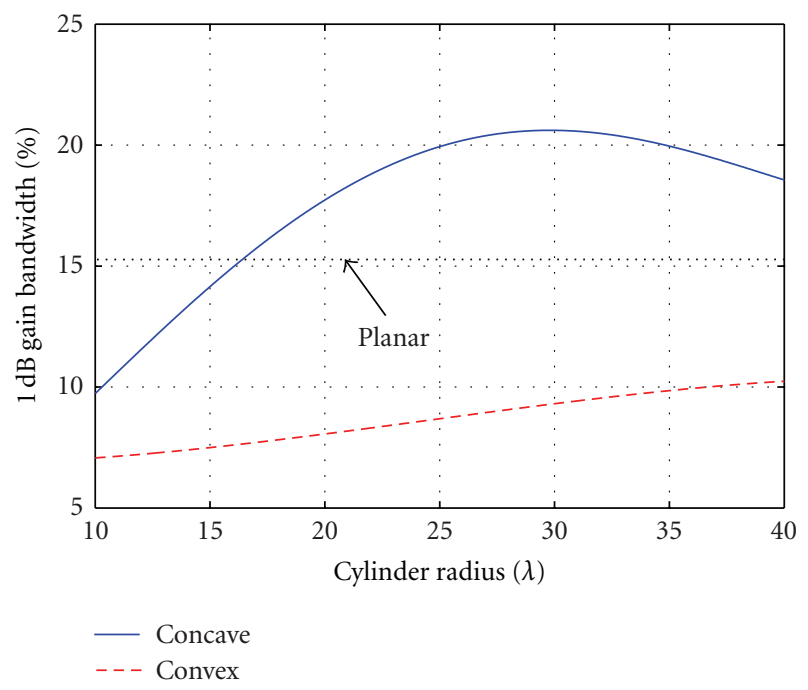

FIGURE 7: Gain bandwidth of the reflectarray antennas as a function of cylinder radius.

the gain bandwidth of a reflectarray antenna can be increased by using a concave cylindrical platform. These studies show that for applications where a slightly curved platform is available, a reflectarray antenna can be a suitable choice for the design.

\section{Acknowledgment}

This work was supported in part by NASA EPSCoR program under the contract number NNX09AP18A.

\section{References}

[1] J. Huang and J. A. Encinar, Reflectarray Antennas, Institute of Electrical and Electronics Engineers, John Wiley \& Sons, 2008.
[2] L. Josefsson and P. Persson, Conformal Array Antenna Theory and Design, IEEE Press Series on Electromagnetic Wave Theory, John Wiley \& Sons, 2006.

[3] A. M. Polegre, G. Caille, L. Boyer, and A. Roederer, "Semiactive conformal array for ESA's GAIA mission," in Proceedings of the IEEE Antennas and Propagation Society Symposium, pp. 4108-4111, Monterey, Calif, USA, June 2004.

[4] J. Huang and R. J. Pogorzelski, "A ka-band microstrip reflectarray with elements having variable rotation angles," IEEE Transactions on Antennas and Propagation, vol. 46, no. 5, pp. 650-656, 1998.

[5] P. Nayeri, F. Yang, and A. Z. Elsherbeni, "Radiation analysis of reflectarray antennas: numerical approaches versus fullWave simulations," in Proceedings of the National Radio Science Meeting, Boulder, Colo, USA, 2012.

[6] R. L. Haupt, Antenna Arrays: A Computational Approach, John Wiley \& Sons, 2010.

[7] R. C. Hansen, Phased Array Antennas, John Wiley \& Sons, 2nd edition, 2009.

[8] Y. Rahmat-Samii, "Reflector antennas," in Antenna Handbook: Theory, Applications, and Design, Y. T. Lo and S. W. Lee, Eds., Van Nostrand Reinhold, 1988.

[9] A. Yu, F. Yang, A. Z. Elsherbeni, J. Huang, and Y. RahmatSamii, "Aperture efficiency analysis of reflectarray antennas," Microwave and Optical Technology Letters, vol. 52, no. 2, pp. 364-372, 2010.

[10] D. M. Pozar, S. D. Targonski, and H. D. Syrigos, "Design of millimeter wave microstrip reflectarrays," IEEE Transactions on Antennas and Propagation, Colorado, U.S., vol. 45, no. 2, pp. 287-296, 1997.

[11] R. J. Mailloux, Phased Array Antenna Handbook, Artech House, 2nd edition, 2005.

[12] D. M. Pozar, "Bandwidth of reflectarrays," Electronics Letters, vol. 39, no. 21, pp. 1490-1491, 2003. 

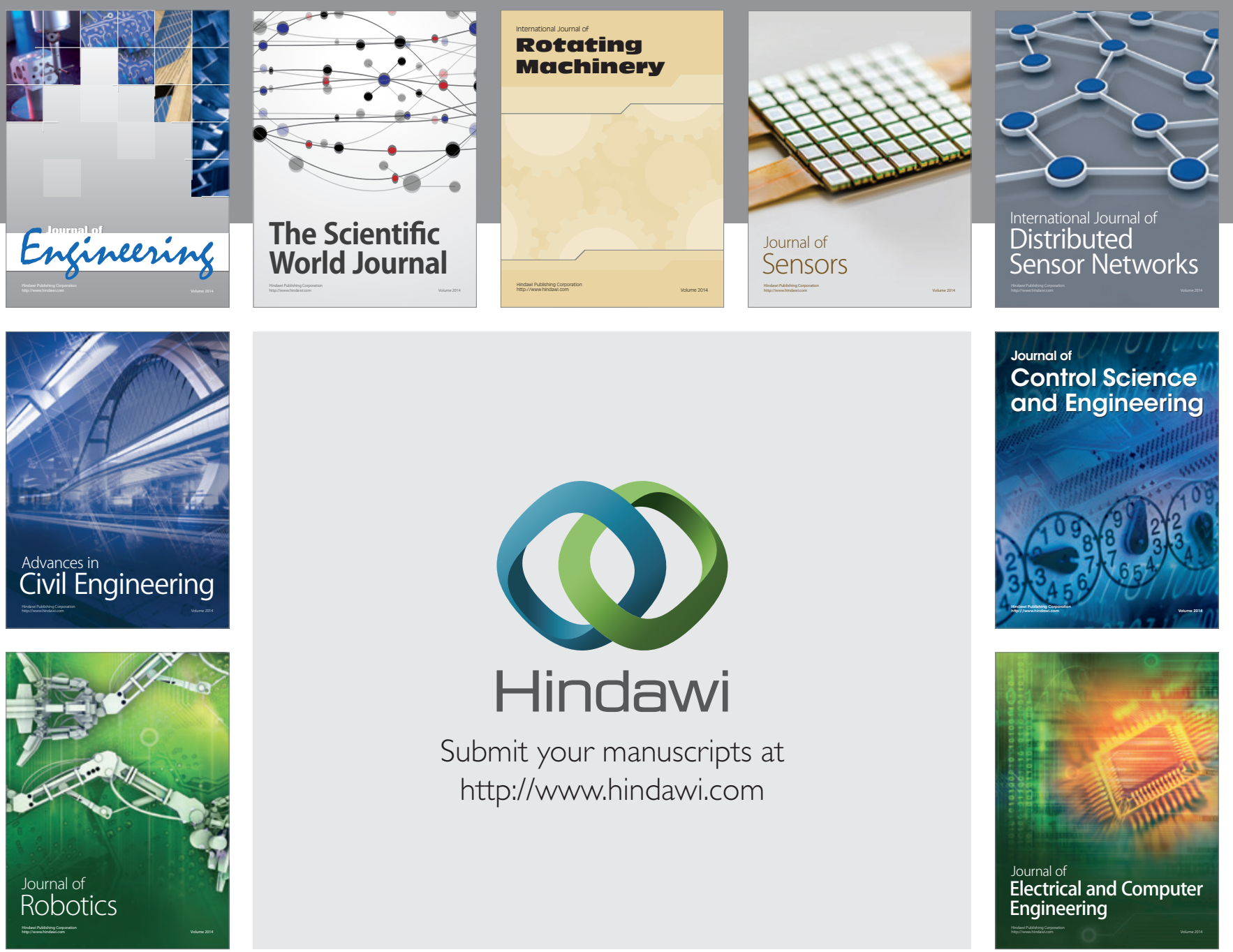

Submit your manuscripts at

http://www.hindawi.com
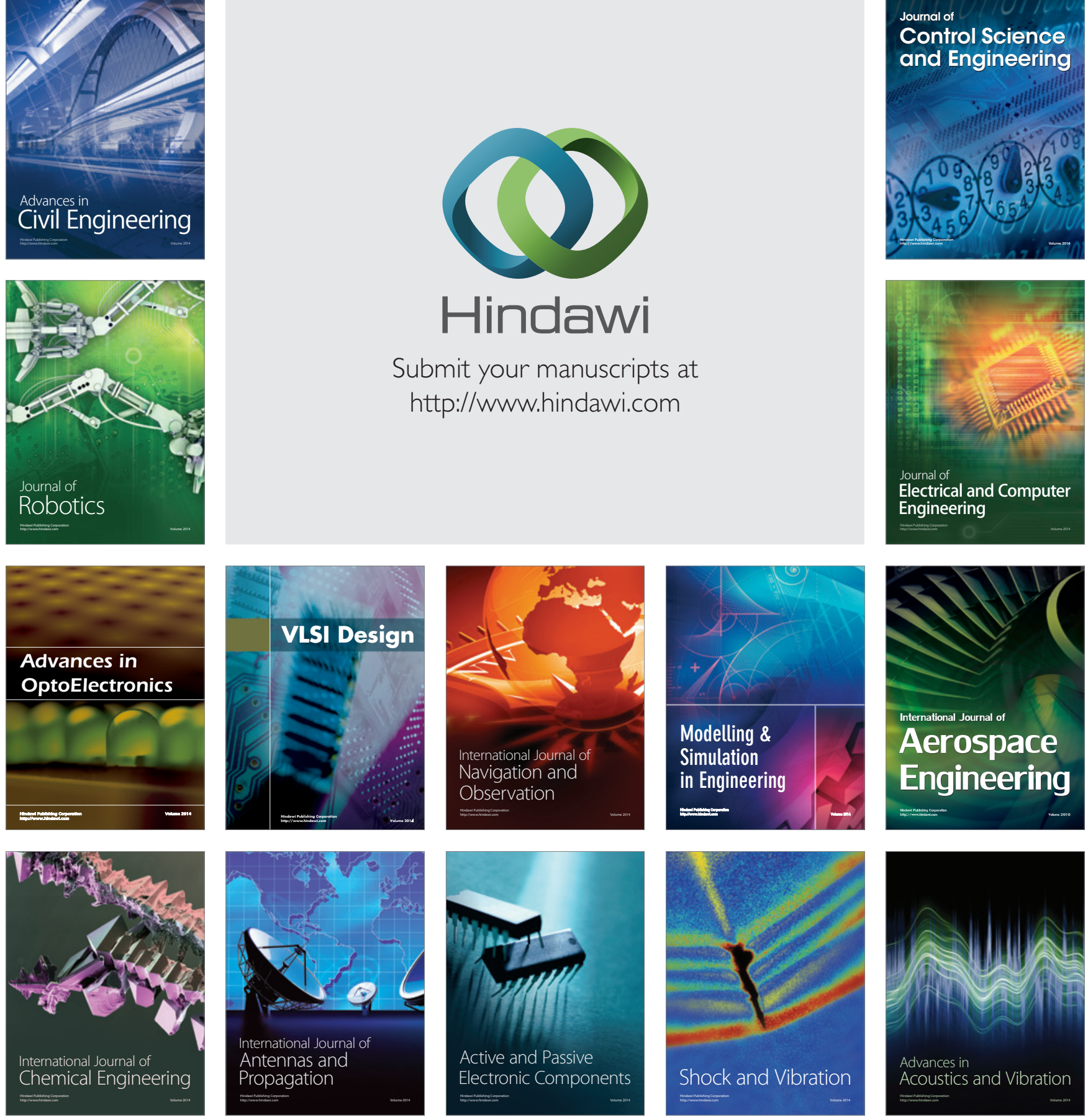